\title{
VLBA Imaging of a Large Sample of Blazars
}

\author{
J. M. Attridge, D. H. Roberts, \& J. F. C. Wardle \\ Department of Physics, Brandeis University, Waltham, MA 02254, U. S. A.
}

\begin{abstract}
As part of our continuing study of parsec-scale magnetic fields in active galactic nuclei we have obtained deep polarization-sensitive images of the blazar $1055+018$ with the VLBA at $5 \mathrm{GHz}$. These dramatic images reveal a magnetized layer of material on the outer surfaces of the jet, distinct from the bulk of the jet. This morphology suggests interaction of the jet with the surrounding medium, the resulting shear stretching the magnetic field lines in the direction of the flow. Further multi-frequency polarization-sensitive observations of $1055+018$ offer the opportunity to study radio jet-ambient medium interactions in detail.
\end{abstract}

\section{Discovery of a Magnetized Sheath in the Blazar $1055+018$}

The optical emission lines and parsec-scale radio jets of active galactic nuclei arise in the same central regions of their host galaxies, and their relationship is a key to understanding these objects. VLBI polarimetry has revealed surprising differences between "strong-" and "weak-lined" sources. The jets of strong-lined sources typically show longitudinal magnetic fields, while those in weak-lined sources are primarily transverse to the jet axis (Cawthorne et al. 1993); however, there are exceptions (e.g., 3C 279; Leppänen, Zensus, \& Diamond 1995; Homan \& Ojha, private communication). To investigate this further we have observed 45 blazars with the VLBA at $5 \mathrm{GHz}$. Of these, four especially interesting sources were chosen for further detailed study. In Figure 1 we show the total intensity and linear polarization distributions of one of these, 1055+018, made from $\sim 4 \mathrm{hr}$ of VLBA+Y1 data taken 20 January 1997. A magnetized "sheath" of material is visible on the outer surfaces of the jet, distinct from the inner jet because the magnetic field in this layer is nearly orthogonal to that within the jet.

A natural interpretation of the jet-sheath structure of $1055+018$ is that transverse shocks dominate within the jet, compressing an initially tangled magnetic field so that the net field is transverse to the jet axis. The sheath is a region in which this same tangled magnetic field is stretched into a longitudinal pattern by a sheared flow arising from jet-confining medium interactions; this field overcomes any transverse field produced by shocks (Wardle et al. 1994).

Future multi-frequency observations of motions in the jet of $1055+018$ will be a direct way to study fluid dynamical processes as the jet plows through the ambient medium. The magnetic field, frozen into the fluid, traces the direction and order of the motions that result. Polarization observations also provide a diagnostic of thermal plasma entrained in the sheath and/or the jet.

Acknowledgments. This work has been supported by the National Science Foundation under grants AST 91-22282, 92-24848, and 95-29228. The National Radio Astronomy Observatory is a facility of the National Science Foundation, operated under a cooperative agreement by Associated Universities, Inc.

\section{References}

Cawthorne, T. V., et al. 1993. ApJ, 416, 519-535.

Leppänen, K. J., Zensus, J. A., \& Diamond, P. J. 1995. AJ, 110, 2479-2492.

Wardle, J. F. C., et al. 1994. $A p J, 437,122-135$. 

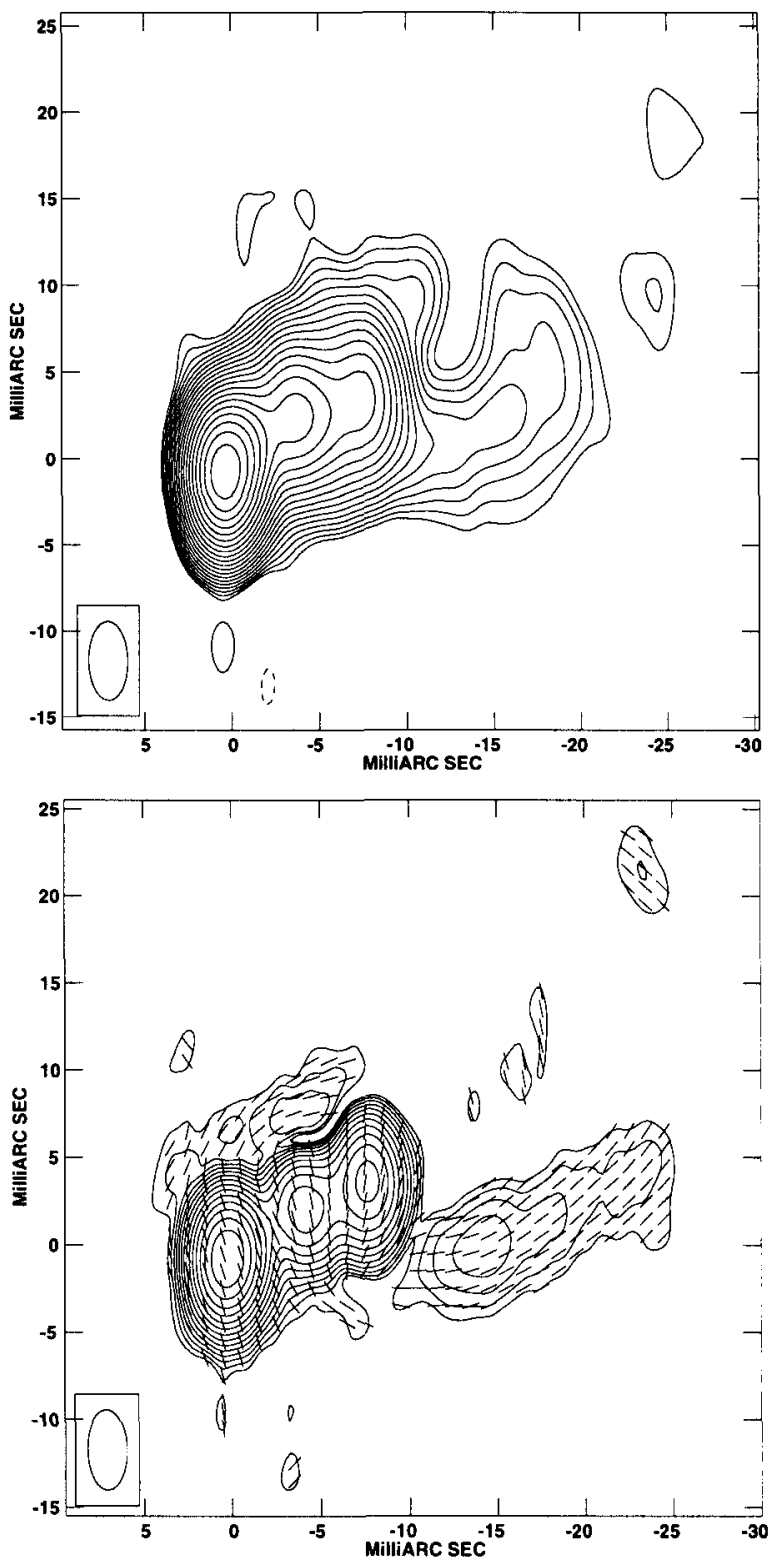

Figure 1. VLBA images of $1055+018$ at $5 \mathrm{GHz}$, epoch 1997.06. (above) Total intensity, with contours at $-1.75,1.75,2.47,3.50,4.95,7.00,9.90,14.0,19.8,28.0$, $39.6,56.0,79.2,112,158,224,317,448,634,896$, and $1270 \mathrm{mJy} /$ beam; the peak is $1.73 \mathrm{Jy} /$ beam. (below) Linear polarization, with contours of polarized intensity at $0.500,0.707,1.00,1.41,2.00,2.83,4.00,5.66,8.00,11.3,16.0,22.6,32.0$, and 45.3 $\mathrm{mJy} /$ beam; the peak is $64.6 \mathrm{~m} \mathrm{Jy} /$ beam. The ticks show the direction of the projected magnetic field, assuming that Faraday rotation (known to be small) is negligible. The $I$ and $P$ images are registered to within a small fraction of a synthesized beamwidth. 